\title{
Optimum SHE for Cascaded H-Bridge Multilevel Inverters Using: NR-GA-PSO, Comparative Study
}

\author{
Basem Alamri 1, Abdulhafid Sallama ${ }^{2}$, Dr. Mohamed Darwish ${ }^{3}$ \\ 'Brunel University, UK, basem.Alamri@brunel.ac.uk , \\ ${ }^{2}$ Brunel University, UK, Abdulhafid.Sallama@brunel.ac.uk, \\ ${ }^{3}$ Brunel University, UK, Mohamed.Darwish@brunel.ac.uk
}

Keywords: Cascaded H-Bridge Multilevel Inverters (CHBMLI), Selective Harmonic Elimination (SHE), NR, GA, PSO.

\begin{abstract}
Selective Harmonic Elimination (SHE) is very widely applied technique in the control of multilevel inverters that can be used to eliminate the low order dominant harmonics. This is considered a low frequency technique, in which the switching angles are predetermined based on solving a system of transcendental equations. Iterative techniques such as NR and Heuristic techniques such as GA and PSO are been ${ }_{\lambda}$ used widely in literatures for the problem of SHE. This paper presents a detailed comparative study of these three techniques when applied for a 7-level CHB-MLI. At the end, several key findings are shared with the readers.
\end{abstract}

\section{Introduction}

Nowadays, multilevel inverters are used in many power industrial and utility applications at different voltage and power levels. The concept of multilevel inverters was introduced back in early 1980s. The term multilevel means that the output voltage waveform composes of three or more steps. The multilevel inverters became very attractive as they produce low harmonic component at low switching frequency. In addition, they result in lower losses, lower blocking voltage of switching devices, and low electromagnetic interference (EMI) [1]. There are basically three main commercial topologies of multilevel voltagesource inverters well placed in industry which are: cascaded H-bridge (CHB-MLI), neutral-point clamped (NPC-MLI) and flying capacitor (FC-MLI). Among these topologies, the CHB-MLI is very widely used in industry for high power applications. It is used in high voltage and high power levels and it requires small number of devices, consequently, it has a reliable modular structure compared to the NPC-MLI and FCMLI.

There are different control techniques which have been proposed and applied for multilevel inverters. These control techniques are classified based on the switching frequency into a) low (fundamental) switching frequency, b) high switching frequency techniques. Space Vector Control (SVC) and Selective Harmonic Elimination (SHE) are low switching techniques in which the active power switch is commutated only one or two times within one cycle. On the other hand, various PWM are used for high switching techniques in which the power switch is switched many times within a cycle [1].

Among different control techniques for multilevel inverters, the SHE is widely used especially for medium voltage and high power applications. The SHE technique has lower switching losses and less EMI because of its low switching compared to other control techniques [2]. In addition, it can eliminate the dominant low order harmonic and hence minimize the size of the required filter at the inverter output. In SHE, the switching angles are pre-calculated based on solving a system of nonlinear equations. In literatures, many techniques and mathematical methods have been proposed and studied for solving the problem of SHE in multilevel inverters. All proposed solution methods can be classified mainly into: a) Iterative techniques, b) Resultant Theory and c) Evolutionary Algorithms.

Among iterative techniques Newton Raphson (NR) is very widely used. In [3-5], NR has been implemented for solving the problem of SHE in multilevel inverts. The method is easy to implement and eliminate the harmonics successfully. However, it does need good initial guess and might not converge at some pints. Other papers [6-7] use resultant theory for SHE. The problem of this techniques in that it gets more complicated with the level increase of the inverter. Another approach in solving the problem of SHE, it to implement Evolutionary Algorithms (EA) such as: GA, PSO, ANN, ...etc. In this approach, the problem of SHE is transformed into constrained optimization problem. In [8-10] GA has been applied for finding the optimum switching angles. PSO is a powerful algorithm in solving SHE and it has been investigated by many researches [11 -12$]$.

In this paper, the most widely used techniques for solving the problem of SHE which are NR, GA and PSO are compared and investigated. The aim of this study is to present a detailed comparison for performance of these techniques in solving the problem of SHE . A 7-level CHB-MLI has been used for this investigation. All simulation and analysis in this research has been conducted using MATLAB-SIMULINK software. 


\section{Cascaded H-Bridge Multilevel Inverters (CHB-MLI)}

The CHB-MLI topology uses a series connection of single phase H-bridge inverters with separate dc sources (SDCS). The main idea is that each bridge (cell) will generate three different voltages and the output waveform can be synthesized by the sum of the voltages generated by each cell Each single phase $\mathrm{H}$-bridge is able to generate $+V_{d c}, 0$ and $-V_{d c}$. This can be obtained by the proper selection of the four switches $S_{1}, S_{2}, S_{3}$ and $S_{4}$ of the cell. This topology has the lowest number of devices when compared to Diode-Clamped (DC-MLI) and Flying Capacitor (FC-MLI). Generally, the separate dc sources are obtained by the use of batteries, fuel cells or solar cells. For reactive power flow pre-charged capacitors are used at the dc side of the inverter [13].

While Diode-Clamped (DC-MLI) and Flying Capacitor (FCMLI) are widely used for industrial medium voltage- high power applications when just low number of levels (typically three) is required, Cascaded H-Bridge inverters (CHB-MLI) are most suitable for high voltage-high power, HVDC utility applications. Mainly due its modular structure which can be extended for high number of levels with no much complexity. Furthermore, with CHB-MLI, higher power and voltage capability can be achieved at lowest number of required devices compared to DC-MLI and FC-MLI. In this paper, a 7 Level CHB-MLI has been chosen to be investigated. The circuit layout for single phase 7-Level CHB-MLI is shown in Figure (1). The switching devices have been selected to be of IGBT type. Each cell is connected to a separate dc link supply of $100 \mathrm{~V}$. Modulation index is changing over a modulation range ( 0 to 1$)$ throughout the analysis. The inverter has been modelled in MALTAL-SIMULINK with main objective of comparing NR, GA and PSO for solving the problem of selective harmonic elimination.

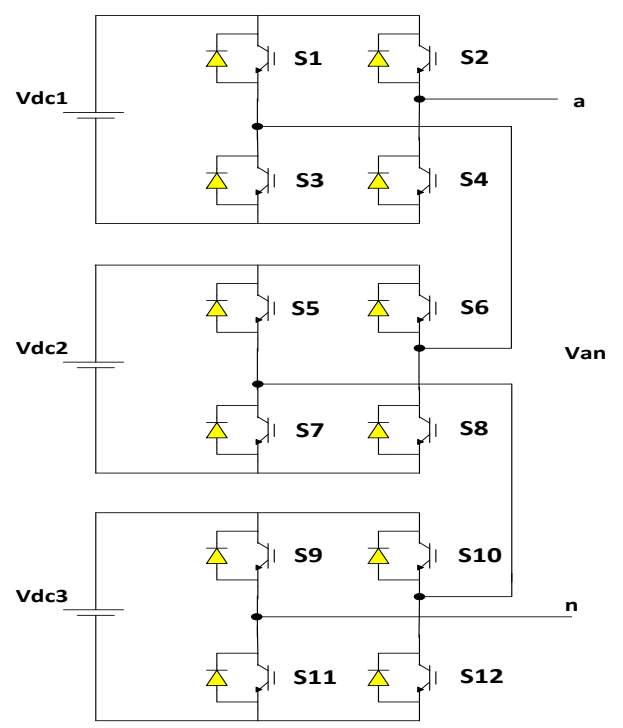

Figure 1: Single-phase 7-level cascaded $\mathrm{h}_{\mathbf{\Lambda}}$-bridge inverter circuit layout.

\section{Selective Harmonic Elimination (SHE)}

To control the output of voltage waveform in multilevel inverters, different modulation techniques have been applied. These control techniques are classified mainly based on the switching frequency into low or high switching techniques. Space Vector Control (SVC) and Selective Harmonic Elimination (SHE) are low switching techniques in which the active power switch is commutated only one or two times within one cycle. On the other hand, various PWM are used for high switching techniques in which the power switch is switched many times within a cycle [1]. Controlling the inverter by SHE will result in less switching losses and less EMI as a result of low switching [2]. In addition, it can eliminate the dominant low order harmonic and hence minimize the size of the required filter at the inverter output. This technique is widely applied for HVDC applications.

SHE uses pre-defined switching angles to form the desired multilevel fundamental voltage and eliminate the predominant low order harmonics which results in minimizing the total harmonic distortion (THD). The switching angles are precalculated off-line and hence this is considered open loop control technique. Figure (2) shows the stepped-voltage waveform for 7-level CHB-MLI. It is clear that, there are 3 switching angles which can be pre-calculated in this case.

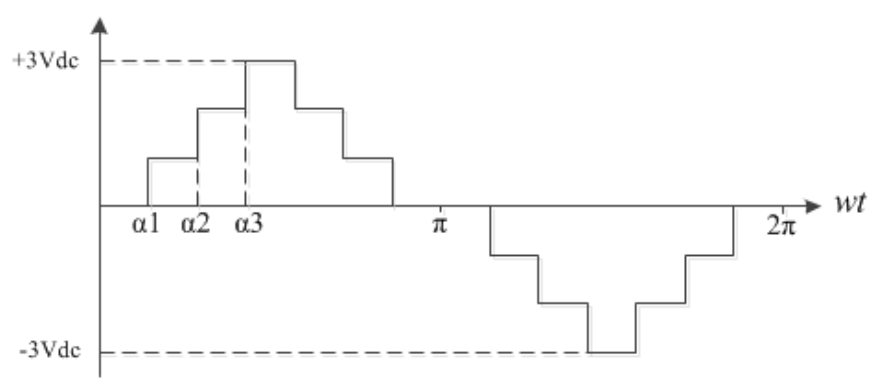

Figure 2: Stepped-voltage waveform for 7-level inverter.

Applying Fourier's expansion, the stepped voltage wave form can be expressed in sum of sine and cosine periodic signals and a constant. The signal consists of odd and even harmonics. Due to the quarter symmetry of the waveform, the even harmonics and the dc constant are cancelled. Hence, only odd harmonics are considered. For balanced three phase systems all triplen harmonics are zero. Generally, The output voltage waveform can be written as:

$$
\begin{aligned}
v_{a n}(w t)= & \sum_{k=1,3,5, \ldots \ldots}^{\infty} \frac{4 V_{d c}}{k \pi}\left[\cos \left(k \propto_{1}\right)\right. \\
& \left.+\cos \left(k \propto_{2}\right) \ldots . .+\cos \left(k \propto_{s}\right)\right] \sin (k w t)
\end{aligned}
$$

Where $(S)$ is the number of H-bride cells of the inverter. 
It is clear from Figure (2) that all switching angles are less than $90^{\circ}$, and are all in ascending order. In 7-level CHB-MLI case,

$$
\theta_{1}<\theta_{2}<\theta_{3}<90^{\circ}
$$

And it is possible to eliminate the $5^{\text {th }}$ and $7^{\text {th }}$ harmonic by solving the following system of non-linear equations where (mi) is the modulation index.

$$
\begin{aligned}
& \cos \left(\theta_{1}\right)+\cos \left(\theta_{2}\right)+\cos \left(\theta_{3}\right)=3 M_{i} \\
& \cos \left(5 \theta_{1}\right)+\cos \left(5 \theta_{2}\right)+\cos \left(5 \theta_{3}\right)=0 \\
& \cos \left(7 \theta_{1}\right)+\cos \left(7 \theta_{2}\right)+\cos \left(7 \theta_{3}\right)=0
\end{aligned}
$$

This system of equations is a highly non-linear. It is also called transcendental equations or SHE equations. To solve such system, different techniques can be applied. Throughout the research papers, Iterative and Evolutionary Algorithms are the most commonly used. The iterative techniques such as Newton-Raphson (NR) have been applied extensively for this problem. Key issue is that when the inverter level gets higher, it becomes more difficult to get to the solution. In addition, it requires good initial guessed values of the switching angles. On the other hand, Evolutionary Algorithms solve the problem using different approach. The main idea is to transform the problem of SHE into optimization problem. The set of transcendental equations will be the constraints for the optimization. In this paper, the problem of SHE for 7-level CHB-MLI is solved using different techniques. The most common algorithms in solving SHE has been investigated in the analysis which are: Newton-Raphson (NR), Genetic Algorithm (GA) and Particle Swarm Optimization (PSO). The results obtained by these techniques are compared and discussed. Matlab -Simulink has been used for the computation and simulation purposes.

\section{Newton Raphson (NR)}

Iterative methods have been used extensively for solving nonlinear equations and locating their roots. Among iterative techniques, the Newton-Raphson is a very common and powerful in solving the problem of transcendental equations. NR is based on linear approximation and applies Taylor's series expansion of the nonlinear function around an initial guess. To find the root of a nonlinear function $f(x)$, an initial estimate (guess) of the root value $x_{0}$ is required to the algorithm. This initial guess should be estimated very carefully. The closest the initial guessed value $x_{0}$ to the real root, the best NR performance will be. On the other hand, NR might be difficult to converge in the case bad initial guessed value.
The first iteration starts based on the value of $x_{0}$.If it does not converge to a feasible solution, the value of the initial guess is further improved by applying the following equation

$$
x_{1}=x_{0}-\frac{f\left(x_{0}\right)}{f^{\prime}\left(x_{0}\right)}
$$

The iterations continue until the stopping criterion of the algorithm satisfied. The estimated value is further improved by the following relation after each iteration

$$
x_{n+1}=x_{n}-\frac{f\left(x_{n}\right)}{f^{\prime}\left(x_{n}\right)}
$$

The NR technique is widely used and applied for the problem of SHE in multilevel inverters. The main drawbacks of this technique are

- Initial guess needs experience and is not easy and has significant effect on the performance of the algorithm.

- It does not converge for some values of modulation index (M). Hence, it is not able to find a solution for the complete range of $(\mathrm{M})$.

Applying NR for solving SHE problem is easy. The following steps summarize and explain the application in the case of 7level CHB-MLI:

STEP_1: Guess initial values of switching angles

$$
\theta^{0}=\left[\begin{array}{lll}
\theta_{1} & \theta_{2} & \theta_{3}
\end{array}\right]^{T}
$$

STEP_2: Evaluate the value of non-linear function matrix at initial guess

$$
F^{0}(\theta)=\left[\begin{array}{c}
\cos \left(\theta_{1}\right)+\cos \left(\theta_{2}\right)+\cos \left(\theta_{3}\right) \\
\cos \left(5 \theta_{1}\right)+\cos \left(5 \theta_{2}\right)+\cos \left(5 \theta_{3}\right) \\
\cos \left(7 \theta_{1}\right)+\cos \left(7 \theta_{2}\right)+\cos \left(7 \theta_{3}\right)
\end{array}\right]
$$

STEP_3: Find the value of the Jacobian matrix using the current guess values.

$$
\frac{d F^{0}(\theta)}{d \theta}=\left[\begin{array}{c}
-\sin \left(\theta_{1}\right)-\sin \left(\theta_{2}\right)-\sin \left(\theta_{3}\right) \\
-5 \sin \left(5 \theta_{1}\right)-5 \sin \left(5 \theta_{2}\right)-5 \sin \left(5 \theta_{3}\right) \\
-7 \cos \left(7 \theta_{1}\right)-7 \sin \left(7 \theta_{2}\right)-7 \sin \left(7 \theta_{3}\right)
\end{array}\right]
$$

STEP_4: Solve for $d \theta$, the value of change in the switching angles

$d \theta^{0}=I N V\left[\frac{d F(\theta)}{d \theta}\right]^{0} X\left(B-F^{0}\right)$

Where B represent the values of required harmonic amplitudes

$$
B=\left[\begin{array}{lll}
3 M & 0 & 0
\end{array}\right]^{T}
$$


STEP_5: Calculate the new switching angles for the next iteration and update their values

$$
\theta^{1}=\theta^{0}+d \theta^{0}
$$

STEP_6: The process is then repeated from 2-5 until the maximum change in the switching angles is less than the desired error. Like all other iterative techniques, it is required for the NR to set a set a condition to stop the algorithm when solving a system of nonlinear equations. Otherwise, the algorithm will not stop in case of no solution exists. The stopping criterion for NR in this problem has been set by satisfaction of either two following conditions:

- $\left|\theta^{n+1}-\theta^{n}\right|$, is sufficiently small and less than the desired tolerance.

- Maximum number of iteration is exceeded , 100 iterations limits considered

Figure (3) explain the flow chart for solving the problem of selective harmonic elimination using Newton-Raphson (NR) method.

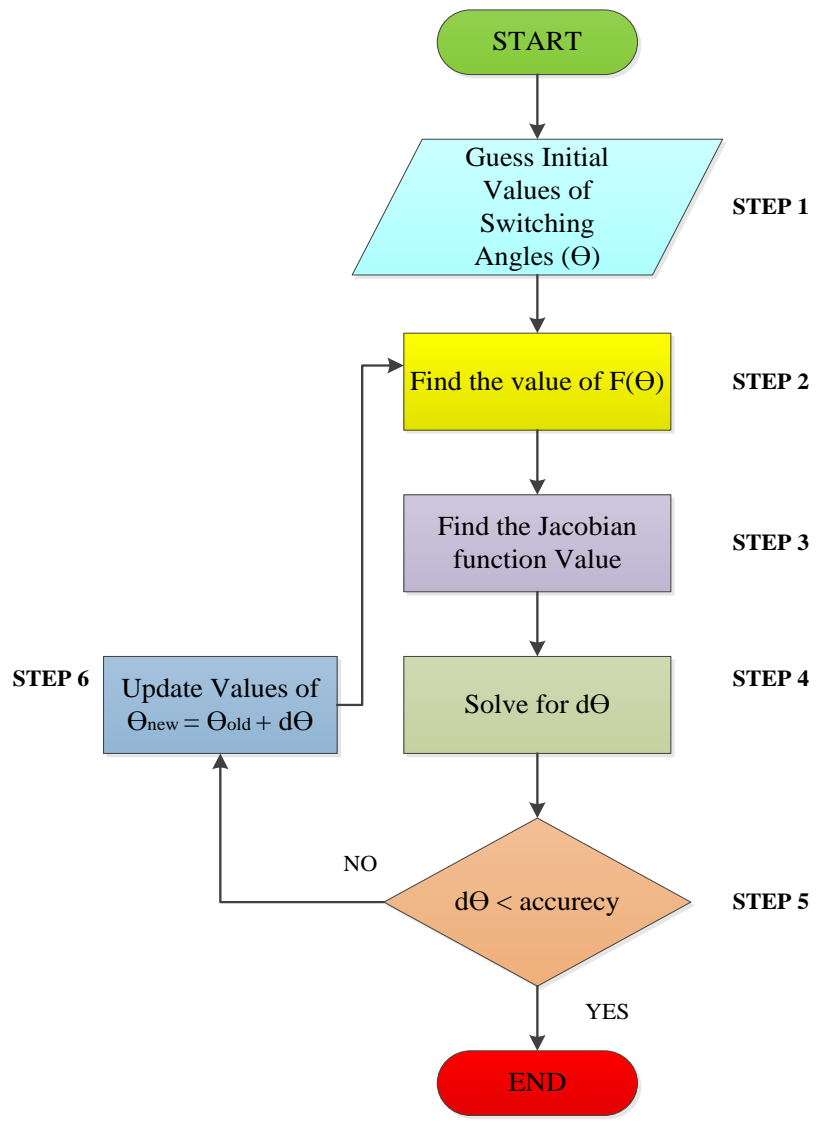

Figure 3: Newton Raphson (NR) for SHE flowchart.

\section{Genetic Algorithm (GA)}

Genetic Algorithm (GA) is a heuristic global evolutionary optimization algorithm which is based on mechanics of natural selection and genetics. The algorithm has initially been developed by John Holland in the early 1970s. This method applies biological evolution in the process of optimization. The key difference compared to other optimization techniques is that GA search by population rather than individual points search. Genetic Algorithm has been widely applied successfully for solving both constrained and unconstrained optimization problems. GA is considered a simple and easy to implement technique which does not include complex derivations or mathematical modelling. Hence, it can be easily applied to solve the problem of selective harmonic elimination.

The process of any Genetic Algorithm optimization consists of four main steps which are: 1) Initialization of the population, 2) Evaluation of fitness function, 3) Selection, and 4) Apply genetic operators. Figure (4) presents a general flow chart for genetic algorithm.

\section{STEP_1: Initialization}

First, the algorithm should be initialized. Parameters of the optimization problem are coded in a binary or floating-point string. Then, a set of solutions is randomly generated based on the coded parameters. This generated set of solutions in called "initial population" $\left(P_{i}\right)$. Each individual feasible solution is considered a chromosome. The number of generated solutions (chromosomes) indicates the "population size". When designing a GA-based optimization for solving the problem of selective harmonic elimination in CHB-MLI, there are $S$ number of switching angles in the solution. Each switching angle is considered a gene. The switching angles (genes) are coded in a binary string as the binary coding system is chosen for the problem. Each chromosome will consist of all genes, $S$ switching angles in this case, where $S$ is the number of cascaded bridges.

$$
\text { Chromosome }=\left[\theta_{1}, \theta_{2}, \cdots \cdots \cdots \cdots, \theta_{S}\right]
$$

Where,

$$
\begin{gathered}
\theta_{1}=[1000] \\
\theta_{2}=[1010] \\
\vdots \\
\theta_{S}=[1100]
\end{gathered}
$$

In this paper, the population size has been chosen to be 30 chromosomes, in which each one consists of 3 genes. The genes of a chromosome are the switching angles $\theta_{1}, \theta_{2}$ and $\theta_{3}$ for the case of 7-level CHB . At the initial population, $\theta_{1}$, $\theta_{2}$ and $\theta_{3}$ are assumed randomly to satisfy the constraints which states that angles should be in ascending order between $0^{\circ}$ and $90^{\circ}$. 


\section{STEP 2: Evaluation of Fitness Function}

In order to test the goodness of a generated solution, a fitness function is to be used as a measure. In this analysis, an objective function has been defined as a fitness value (FV). The fitness function should be determined very carefully as it has a great effect on the quality of the optimization result.

In the case of 7-level CHB selective harmonic elimination problem, the $5^{\text {th }}$ and the $7^{\text {th }}$ harmonic are to be eliminated. Furthermore, the fundamental waveform is to be as desired Hence, the difference between the fundamental and the reference output voltage should be minimized. Thus, an objective function was defined to meet the above requirement and at the same time minimize the THD of the output waveform.

$$
O b j_{-} \text {Fun }=\left|V_{1}-S M_{i}\right|^{4}+\left|V_{5}\right|^{2}+\left|V_{7}\right|^{2}+\% T H D
$$

Where $V_{1}, V_{5}$ and $V_{7}$, are the amplitudes of fundamental, $5^{\text {th }}$ and $7^{\text {th }}$ harmonics respectively. $S$ is the number of cascaded h-bridges which is 3 in this case

\section{STEP_3: Selection}

At selection stage, parents are chosen based on selection rules to produce offspring chromosomes. The selected parents are the main contributors to form the next generation. In this the fittest individual are likely to survive and the less fit are eliminated.

\section{STEP_4: Crossover and mutation}

Crossover is a genetic operator applied, in which a number of bits are swapped between parents. Basically in crossover some genes are exchanged to form a new improved combination. Crossover is considered a very important and a powerful genetic operator. Another, operator to be applied at low probability is called Mutation, in which the genes are alerted. This can be accomplished by changing a bit within a gene from 0 to 1 or from 1 to 0 . Mutation expands the search space and hence prevents the algorithms from falling into local minimum.

\section{STEP_5: Stopping Criterion}

Deciding the stopping criterion is of significant importance in any GA-based optimization problem. Basically, it tells the algorithm when to stop and terminate. Hence, it decides the optimum solution as an output. The algorithm should stop after 100 iterations are performed. In some cases, the algorithm finds a solution much time before 100 iterations. In this case, the algorithm should stop when the weighted average change in the fitness function over 50 iterations is less than a function tolerance $1 \times 10^{-6}$, in this analysis.

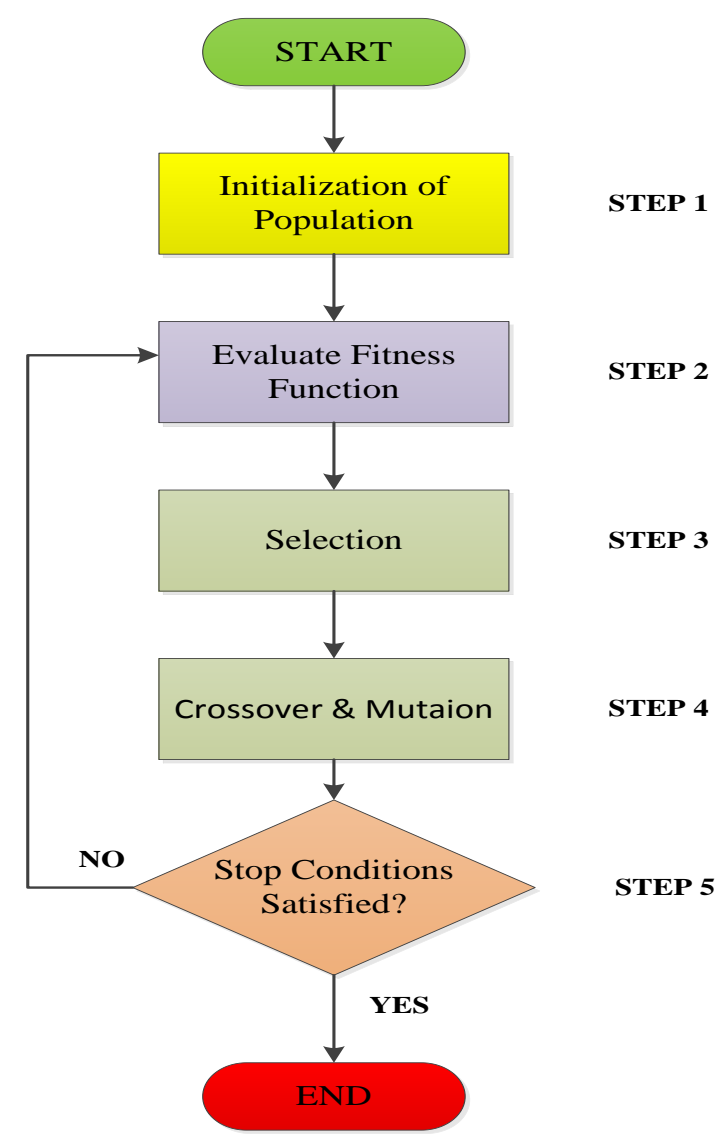

Figure 4: General GA algorithm flowchart.

\section{Particle Swarm Optimization (PSO)}

Particle swarm optimization (PSO) was developed as a simulation of the bird flocking flow [14], in two space dimensions $(\mathrm{x}, \mathrm{y})$, where $(\mathrm{vx})$ represents the agent velocity in the direction of $\mathrm{x}$-axis, (vy) represents the agent velocity in the direction of $y$-axis, $(x, y)$ represents the agent's current position and (vx, vy) represents the current velocities in two dimensions. From the velocity and position information, the agent can be modified for the new position.

The school of fish and birds flock optimises a given objective function based on the experiences and every time solution. In this case study, the objective function was given to the PSO algorithm is the same given to the GA algorithm which is shown in equation (15). The particle recognizes this information and an analogy is stored each time in under the name of the local best solution (pbest). At the same time in every cycle all particles recognize the best solution for all groups stored each time under the name of the global best solution (gbest). Depending on this information, each particle recognizes its performance, and performs in tandem with all other particles in the group. Therefore, each particle tries to adjust its position. 
This is demonstrated in Figure (5) using the following information:

- Current positions (x, y),

- Current velocities (vx, vy),

- Distance between the current position $(\mathbf{x}, \mathbf{y})$ and pbest

- Distance between the current position $(\mathbf{x}, \mathbf{y})$ and gbest

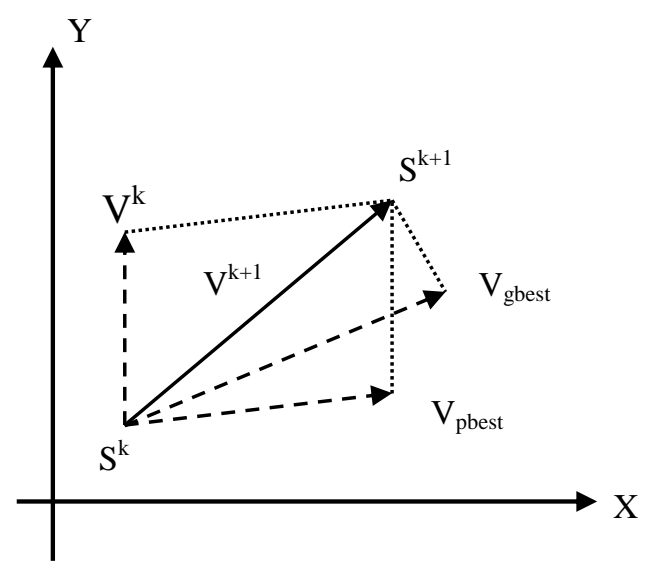

Figure 5: Concept of a modification of a searching point by PSO

Where

$\mathbf{S}^{\mathbf{k}}$ : current searching point.

$\mathbf{S}^{\mathbf{k}}+\mathbf{1}$ : modified searching point.

$\mathbf{V}^{\mathbf{k}}$ : current velocity.

$\mathbf{V}^{\mathbf{k}+\mathbf{1}}$ : modified velocity.

Vpbest: velocity based on pbest.

Vgbest: velocity based on gbest

From the concept of velocity the new position is represented and modified (i.e. the modified value for the current positions). The following equation (16) expresses the modified velocity of each particle:

$$
\begin{aligned}
& V_{i}^{k+1}=W V_{i}^{k}+c_{1} \times \operatorname{rand}_{1} \times\left(\text { pbest }_{i}-S_{i}^{k}\right) \\
& +c_{2} \times \text { rand }_{2} \times\left(\text { gbest }_{i}-S_{i}^{k}\right)
\end{aligned}
$$

Where

$\mathbf{V}_{i}^{\mathbf{k}+\mathbf{1}}$ : velocity of particle $\boldsymbol{i}$ at iteration $\mathbf{k + 1}$.

$\mathbf{V}_{i}^{\mathbf{k}}$ : velocity of particle $\boldsymbol{i}$ at iteration $\mathbf{k}$.

$\mathbf{W}$ : inertia function.

C1 and C2: are the acceleration constants.

Rand1 and Rand2: random number between 0 and 1 .

$\mathbf{S}_{\boldsymbol{i}}^{\mathbf{k}:}$ current position of particle $\boldsymbol{i}$ at iteration $\boldsymbol{k}$.

pbest $_{i}$ : best position of particle $\boldsymbol{i}$.

gbest: the global best position of the group.
While the inertia weighting function is usually utilized as follows:

$W=W_{\max }-\frac{W_{\max }-W_{\min }}{\text { iter }_{\max }} \times$ iter $_{i}$

Where

$\boldsymbol{W}_{\text {max }}$ : initial weight,

$W_{\text {min }}$ : final weight,

iter $_{\text {max }}$ : maximum iteration number,

iter $_{i}$ : current iteration number.

Equation (16) can be explained as follows. The RHS of consists of three terms, the first of which is the previous velocity of the particle. The second and third terms are utilized to change the velocity of the particle. Without the second and third terms, the agent will keep on "flying" in the same direction until it hits the boundary (i.e. it tries to explore new areas). Therefore, the first term corresponds to the diversification in the search procedure. On the other hand, without the first term, the velocity of the "flying" particle is only determined by using its current position and its best positions pbest in history. The particles will try to converge in the pbests and/or gbest, therefore the terms are corresponding to intensification in the search procedure [15-16].

Figure (5) illustrates a concept of modification with particles in the solution space. Each particle finds the new position using the integration of velocity vectors. The current position can be modified by the following equation:

$$
S_{i}^{k+1}=S_{i}^{k}+V_{i}^{k+1}
$$

Where

$\mathrm{S}^{\mathrm{k}+1}$ : modified searching point.

$\mathrm{S}^{\mathrm{k}}$ : current searching point.

$\mathrm{V}^{\mathrm{k}+1}$ : modified velocity.

\subsection{PSO Algorithm}

PSO algorithm comprises a very simple concept, and paradigms are implemented in a few lines of computer code. The general flow chart of PSO is shown in Figure (6) and each step is explained here below:

STEP_1: Generate initial condition for each agent. Initial position searching points of particle $\boldsymbol{i}$ at iteration $\boldsymbol{k}=\mathbf{0},\left(\mathbf{S}_{\boldsymbol{i}}^{\mathbf{k}}\right)$ and velocities $\left(\mathbf{V}_{i}^{\mathbf{k}}\right)$ of each agent are usually generated randomly within the allowable range. The current searching point is set to pbest for each agent. The best-evaluated value of pbest is set to gbest and the agent number with the best value is stored. 


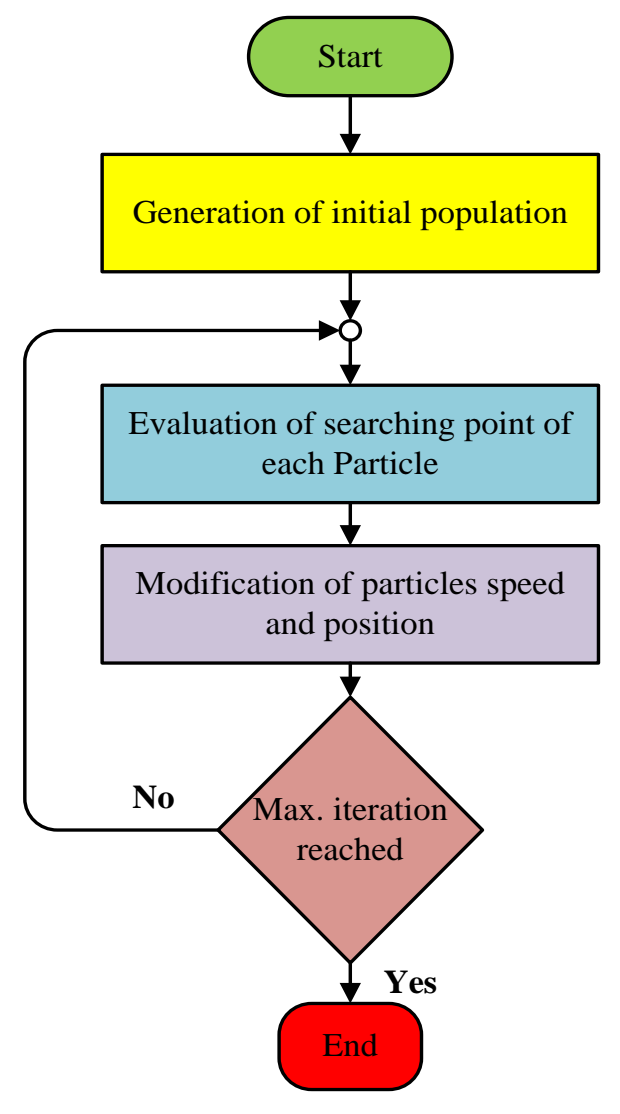

Figure 6: General PSO algorithm flowchart.

STEP_2: Evaluation of searching point of each particle. The objective function value is calculated for each particle. If the obtained value is better than the current local best value pbest of the particle, the new pbest value is replaced by the current value. If the local best value of pbest is better than the current global best value gbest, then the new gbest is replaced by the best value and the particle number with the best value is stored.

STEP_3: Modification of each searching point $S^{k+1}$. The current searching point $S^{\mathrm{k}}$ of each particle updated using previous equations (16) and (18).

STEP_4: Checking the exit condition. The current iteration number reaches the predetermined maximum iteration number, then exit. Otherwise, go to step 2.

The detailed features of the searching procedure of PSO are:

- As shown in equations (16) and (18), PSO can essentially handle continuous optimization problem.

- Similar to GA, the PSO utilizes several searching points and the searching points gradually get close to the optimal point using their local best pbests and the global best gbest.
- The first term of right-hand side at equation 1 corresponds to diversification in the search procedure. The second and third terms at RHS of the equation correspond to intensification in the search procedure. This method has a well-balanced mechanism to utilize diversification and intensification in the search procedure efficiently.

- The above concept of PSO can use more than two dimensions in the space. However, the method can be easily applied to n-dimension problem. In other words, PSO can handle continuous optimization problems with continuous state variables in an $\mathrm{n}$-dimension solution space.

\section{Simulation Results}

This section presents the simulation results obtained and compare the three approaches under study: NR, GA and PSO for solving the problem of SHE. These simulation results is for 7-level CHB-MLI and it covers complete range of modulation index $\left(M_{i}=0.05\right.$ to 1.0$)$. The simulation shows that NR method was not able to find a solution for the complete range of modulation index. Actually, NR does find a solution for the modulation index range $\left(M_{i}=0.3\right.$ to 0.9$)$. Even within this range, it was not possible for NR to find a feasible solution for every point of $M_{i}$. On the other hand, both GA and PSO were capable to solve the problem of SHE over the complete range of modulation index $\left(M_{i}=\right.$ 0.05 to 1.0) and they can find a solution for all points within this range for the problem under investigation. While NR was good in only eliminating the selected harmonics, GA and PSO were capable of minimizing the THD as well in addition to eliminating the harmonics. The simulation results show that GA and PSO are much better in terms of minimizing the THD compared to NR. Actually, GA gives the lowest THD compared to PSO especially for the lower modulation index range $\left(M_{i}=0.05\right.$ to 0.6$)$. Figure (7) present a comparison of THD obtained for different modulation index values using NR, GA and PSO. It is clear that NR has the worst THD profile and GA results in minimum THD. Figure (8) present the THD for each solving method separately. In this figure, the coloures denote the different values of modulation index.

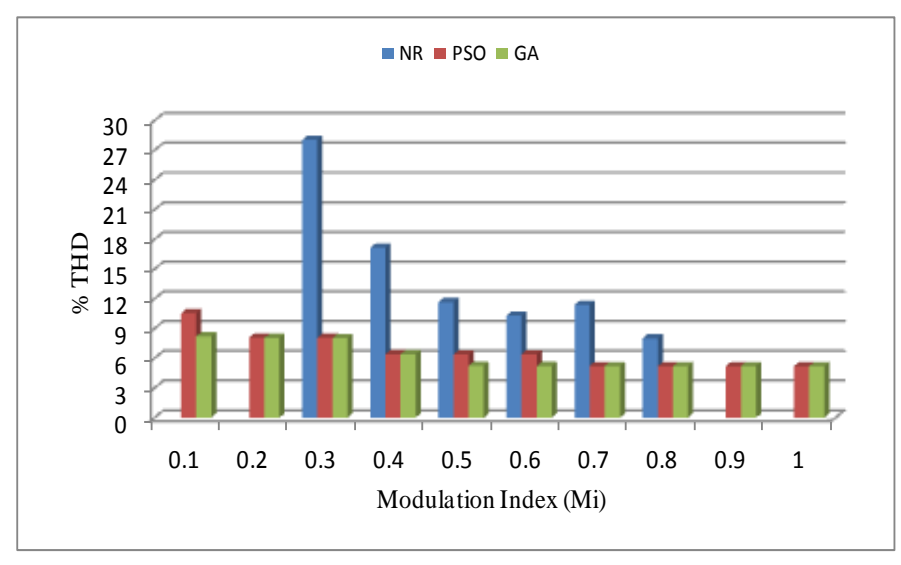

Figure 7: THD at different modulation index values. 


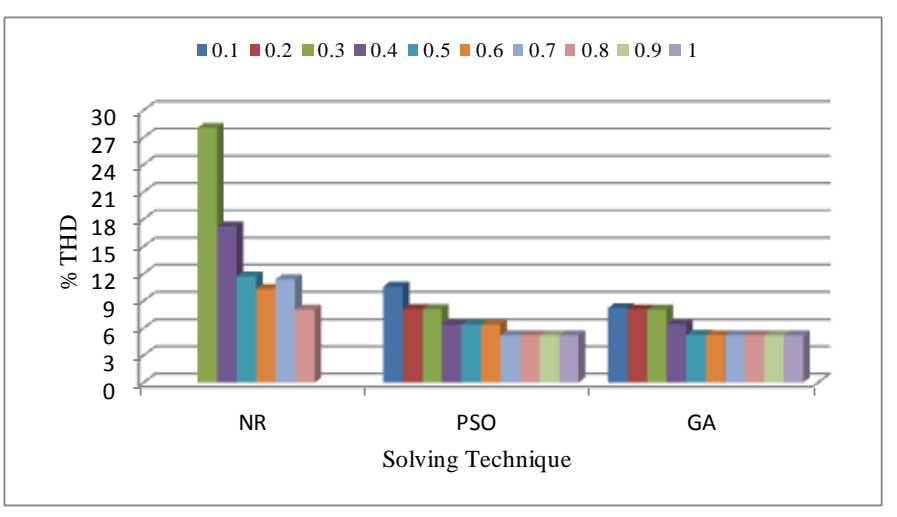

Figure 8: THD profile for each solving technique.

In Table (1), the detailed calculations of switching angles and THD at each modulation index value are demonstrated. The ** indicates that the algorithm failed to find a feasible solution at that point of $M_{i}$. The switching angles obtained using NR, GA and PSO are shown in Figures (9), (10) and (11) respectively.

The results show that NR solution switch at higher switching angles compared to GA and PSO. The table shows clearly that GA has the best performance in terms of THD. The NR does not converge to a feasible solution over the complete range $M_{i}$.

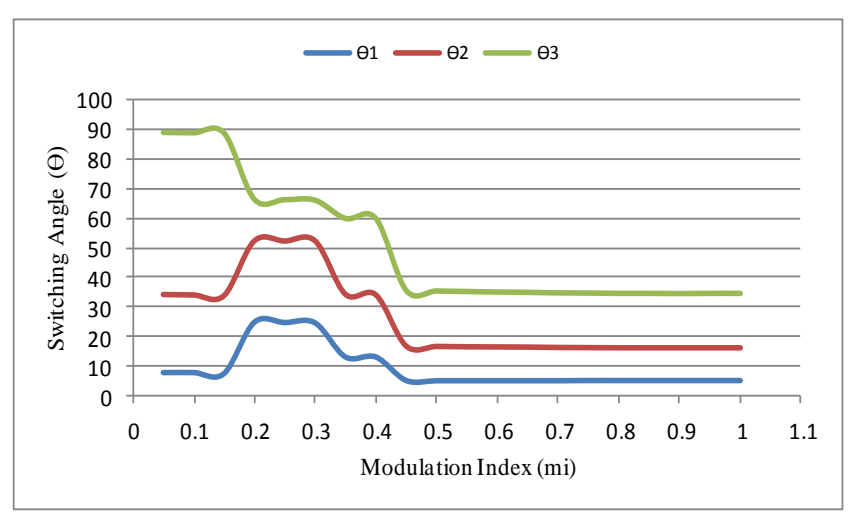

Figure 9: Switching angles using NR.

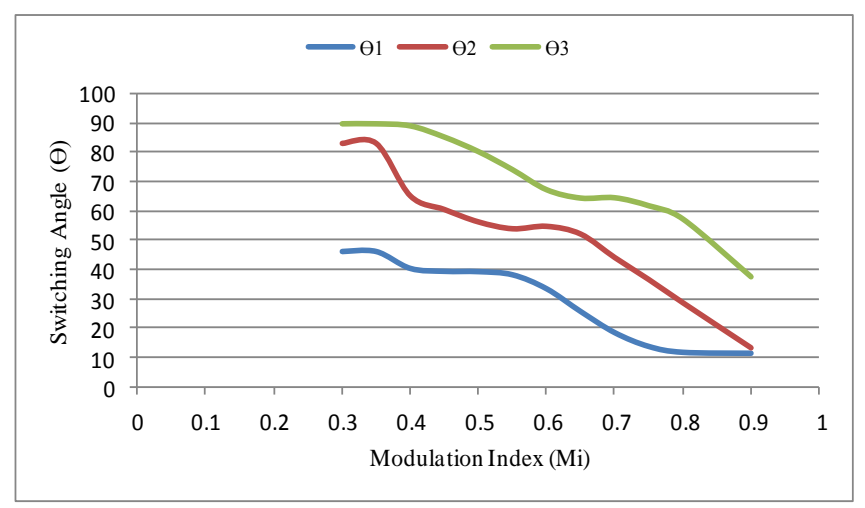

Figure 10: Switching angles using GA

\begin{tabular}{|c|c|c|c|c|c|c|c|c|c|c|c|c|}
\hline \multirow{2}{*}{ Mi } & \multicolumn{3}{|c|}{$\theta 1$} & \multicolumn{3}{|c|}{$\theta 2$} & \multicolumn{3}{|c|}{$\theta 3$} & \multicolumn{3}{|c|}{$\%$ THD } \\
\hline & NR & PSO & GA & NR & PSO & GA & NR & PSO & GA & NR & PSO & GA \\
\hline 0.05 & $* *$ & 39.2 & 8.0 & $* *$ & 59.6 & 34.4 & $* *$ & 81.3 & 89.0 & $* *$ & 10.6 & 8.2 \\
\hline 0.1 & $* *$ & 39.3 & 8.0 & $* *$ & 59.6 & 34.3 & $* *$ & 81.1 & 88.9 & $* *$ & 10.5 & 8.2 \\
\hline 0.15 & $* *$ & 39.3 & 7.7 & $* *$ & 59.5 & 34.0 & $* *$ & 81.0 & 88.8 & $* *$ & 10.5 & 8.1 \\
\hline 0.2 & $* *$ & 7.6 & 24.7 & $* *$ & 34.0 & 52.5 & $* *$ & 88.7 & 66.5 & $* *$ & 8.1 & 8.0 \\
\hline 0.25 & $* *$ & 7.6 & 24.6 & $* *$ & 33.9 & 52.5 & $* *$ & 88.7 & 66.3 & $* *$ & 8.1 & 8.0 \\
\hline 0.3 & 46.4 & 7.6 & 24.5 & 83 & 33.9 & 52.5 & 89.6 & 88.6 & 66.2 & 28.1 & 8.1 & 8.0 \\
\hline 0.35 & $* *$ & 7.5 & 13.2 & $* *$ & 33.8 & 34.5 & $* *$ & 88.6 & 60.0 & $* *$ & 8.1 & 6.4 \\
\hline 0.4 & 40.5 & 13.2 & 13.2 & 65.1 & 34.3 & 34.3 & 88.9 & 60.0 & 60.0 & 17.2 & 6.4 & 6.4 \\
\hline 0.45 & 39.5 & 13.2 & 5.4 & 60.5 & 34.2 & 17.0 & 85.1 & 59.9 & 35.8 & 13.6 & 6.4 & 5.3 \\
\hline 0.5 & 39.4 & 13.1 & 5.3 & 56.3 & 34.1 & 16.9 & 80.1 & 59.9 & 35.6 & 11.7 & 6.4 & 5.2 \\
\hline 0.55 & 38.3 & 13.1 & 5.3 & 53.9 & 34.0 & 16.8 & 73.9 & 59.9 & 35.5 & 12.2 & 6.4 & 5.2 \\
\hline 0.6 & 33.5 & 13.1 & 5.3 & 54.8 & 34.0 & 16.7 & 67.1 & 59.9 & 35.3 & 10.3 & 6.4 & 5.2 \\
\hline 0.65 & 25.6 & 5.3 & 5.3 & 52.1 & 16.6 & 16.7 & 64.3 & 35.2 & 35.2 & 8.9 & 5.2 & 5.2 \\
\hline 0.7 & 18.3 & 5.3 & 5.3 & 44.1 & 16.6 & 16.6 & 64.4 & 35.0 & 35.0 & 11.4 & 5.2 & 5.2 \\
\hline 0.75 & 13.5 & 5.4 & 5.4 & 36.6 & 16.5 & 16.5 & 61.6 & 34.9 & 35.0 & 7.8 & 5.2 & 5.2 \\
\hline 0.8 & 11.5 & 5.4 & 5.4 & 28.7 & 16.5 & 16.4 & 57.1 & 34.8 & 34.8 & 8.0 & 5.2 & 5.2 \\
\hline 0.85 & $* *$ & 5.4 & 5.4 & $* *$ & 16.4 & 16.4 & $* *$ & 34.8 & 34.8 & $* *$ & 5.2 & 5.2 \\
\hline 0.9 & 11.2 & 5.4 & 5.4 & 13.4 & 16.4 & 16.4 & 37.4 & 34.8 & 34.8 & 9.2 & 5.2 & 5.2 \\
\hline 0.95 & $* *$ & 5.4 & 5.4 & $* *$ & 16.4 & 16.4 & $* *$ & 34.8 & 34.8 & $* *$ & 5.2 & 5.2 \\
\hline 1 & $* *$ & 5.4 & 5.4 & $* *$ & 16.4 & 16.4 & $* *$ & 34.8 & 34.8 & $* *$ & 5.2 & 5.2 \\
\hline
\end{tabular}

Table 1: Detailed calculation of switching angles and THD obtained using different solving techniques. 


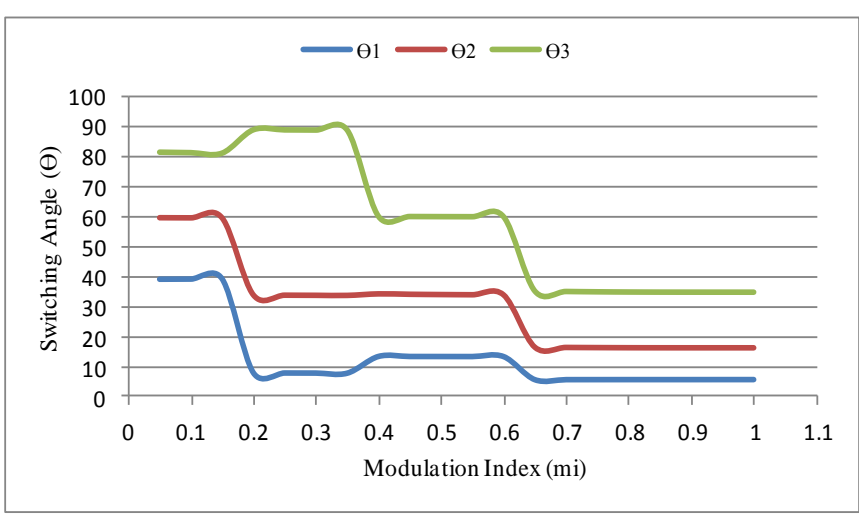

Figure 11: Switching angles using PSO.

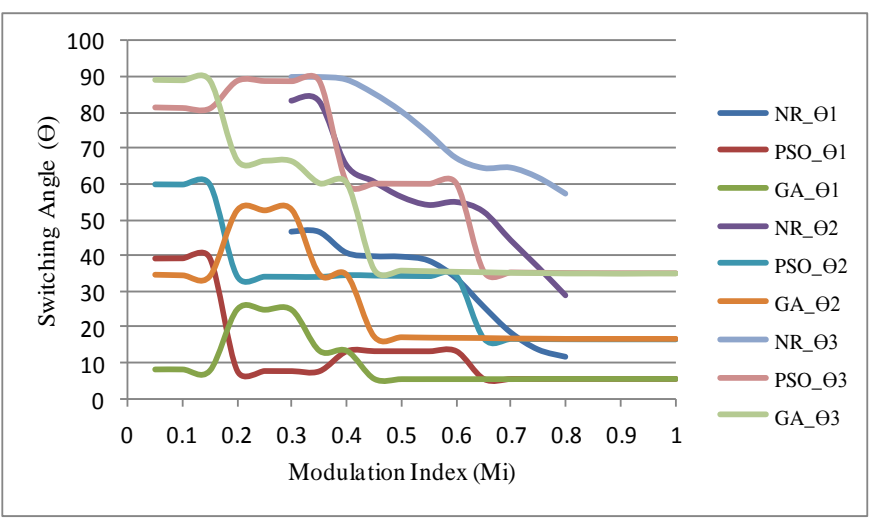

Figure 12: Switching angles using NR-GA-PSO.

Figure (12) compares all calculated switching angles obtained by NR, GA and PSO. It is clear that GA and PSO gives same switching angles for $\left(M_{i}=0.65\right.$ to 1.0$)$. The harmonic profile is compared in Figure(13). For the $5^{\text {th }}$ harmonic, NR has the lowest value and PSO has the highest compared to other methods. While for the $7^{\text {th }}$ harmonic GA has the lowest value and then NR. PSO still has the highest value for the $7^{\text {th }}$ harmonic as for the $5^{\text {th }}$. Looking for the whole harmonic spectrum, one can conclude that while GA technique has the best performance for SHE, NR technique has the worst performance among the three compared techniques.

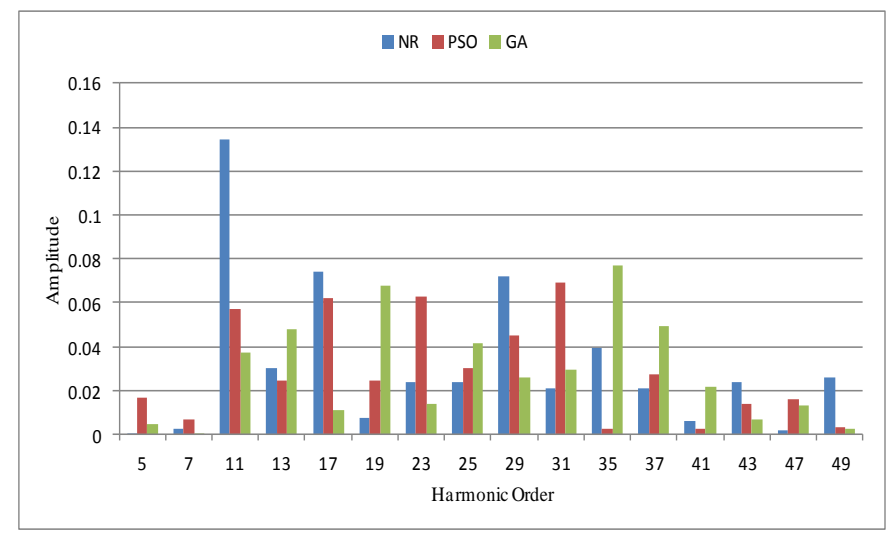

Figure 13: Harmonic profile using NR-GA-PSO.

\subsection{Key Findings and Conclusive Remarks}

- SHE problem is based on solving a set of transcendental equations.

- Iterative techniques such as NR can be implemented easily.

- NR does need a good initial guess for the switching angles. This guess affects the performance of NR in solving the problem significantly.

- NR might not converge at some points within the range of $\mathrm{Mi}$, and hence it is not suitable for applications when a complete range of $\mathrm{Mi}$ is required.

- NR is getting more complicated and difficult to converge at higher levels inverters.

- GA and PSO transform the problem of SHE into optimization problem.

- It is very crucial in optimization to define the objective function properly.

- In GA and PSO, no initial guess is required.

- Both GA and PSO find a solution of SHE problem over the complete range of Mi.

- For this case study, GA was found to be the best solving method in terms of harmonic profile and THD.

- In terms of eliminating the $5^{\text {th }}$ and $7^{\text {th }}$ harmonics, PSO was the worst with having the highest amplitude for these harmonics which is required to be eliminated.

\section{Conclusion}

The problem of Selective Harmonic Elimination (SHE) in multilevel inverters has been investigated in this paper. Different important solving approaches which are: NR, GA and PSO have been considered. The properties of each approach was carefully explained and employed for SHE in 7-level cascaded h-bridge. It has been shown that it possible to transform the problem of SHE into an optimization problem and heuristic algorithms can be implemented easily for the solving the problem. The study concludes that Evolutionary Algorithm such as GA and PSO are very powerful in solving SHE and results in considerable reduction in the THD compared to iterative techniques. In this case, GA was found to be better than PSO especially at low $M_{i}$ values. The most crucial part when using EA for solving SHE is to properly define the objective function. 


\section{References}

[1] N. Mittal, B. Singh, S.P. Singh, R. Dixit and D. Komar, "Multi-level inverter: a literature survey on topologies and control strategies", ICPCES, $2^{\text {nd }}$ International Conference on Power, Control and Embedded Systems, 2012.

[2] M.G.H. Aghdam, S.S. Fathi and A. Ghasemi, "The analysis of conduction and switching losses in threephase OHSW multilevel inverter using switching functions", IEE PEDS 2005, Vol. 1, pp. 209-218, 2005.

[3] Jagdish Kumar, Biswarup Das and Pramod Agarwal, "selective harmonic elimination technique for a multilevel inverter", $15^{\text {th }}$ National Power System Conference (NPSC), December 16-18, 2008, paper no. 168, pp. 608-613, IIT Bombay, December, 2008.

[4] Jagdish Kumar, "THD analysis for different levels of cascaded multilevel inverters for industrial applications", International Journal of Emerging Technology and Advanced Engineering (IJETAE), Vol. 2, Issue 10, pp.237-244, October 2012

[5] Gobinath K, Mahendran S and Gnanambal I, "New cascaded h-bridge multilevel inverter with improved efficiency", International Journal of Advanced Research in Electrical, Electronics and Instrumentation Engineering (IJAREEIE), Vol. 2, Issue 4, pp.1263-1271, April 2013

[6] John C, Leon Tolbert, Keith $\mathrm{M}$ and Zhong D, "A new approach to solving the harmonic elimination equations for a multilevel converter", in Proc. IEEE Industry Applications Soc. Annu. Meeting, Salt Lake City, UT, pp. 640-645, October 2003.

[7] Zhong D, Leon M and John C, "Active Harmonic Elimination for Multilevel converters", IEEE Trans. Power Electronics, Vol. 21 , Ni. 2, pp. 459-469, March 2006.

[8] Burak O, Leon M and John C, “ Harmonic optimization of multilevel converters using genetic algorithm", IEEE Power Electronics Letters, Vol.3, No. 3, pp.92-95, September 2005

[9] MAruthu Pandi P, Devarajan N, “ Performance enhancement of embedded system based multilevel inverter using genetic algorithm", Journal of Electrical Engineering, Vol. 62, No. 4, pp. 190-198, 2011

[10] Darshan K, Swapnajit P and Varsha S, "Genetic Algorithm Based Approach for Optimization of conducting angles in cascaded multilevel inverter", International Journal of Engineering Research and Applications (IJERA), Vol. 2, Issue 3, pp.2389-2395, May-June 2012
[11] N. Prashanth, B. Kumar, J. Yadagiri, A.Dasgupta, "Harmonic minimization in multilevel inverters by using PSO", ACEEE Int. J. on Control System and Instrumentation, Vol. 02, No. 03, October 2011

[12] T. Jeevabharath and V. Padmathilagam, "Harmonic elimination of cascaded multilevel inverters using particle swarm optimization”, International Conference on Computing and Electrical Technologies (ICCEET), pp.301-306, Kumaracoil, 21-22 March, 2012

[13] C. Govindaraju1 and K. Baskaran, "Performance improvement of multiphase multilevel inverter using hybrid carrier based space vector modulation", International Journal on Electrical Engineering and Informatics - Volume 2, Number 2, 2010,pp 137-149.

[14] J. Kennedy, J. F. Kennedy and R. C. Eberhart, Swarm Intelligence. Morgan Kaufmann, 2001.

[15] H. Schwefel, Numerical Optimization of Computer Models. John Wiley \& Sons, Inc., 1981.

[16] Sallama, Abdulhafid, Maysam Abbod, and Shariq Mahmood Khan. "Applying sequential particle swarm optimization algorithm to improve power generation quality", International Journal of Engineering and Technology Innovation, 2014

[17] Alamri, Basem, and Mohamed Darwish. "Precise modelling of switching and conduction losses in cascaded h-bridge multilevel inverters" In Power Engineering Conference (UPEC), 2014 49th International Universities, pp. 1-6. IEEE, 2014. 